\title{
Soil properties from low-velocity probe penetration
}

\author{
Jerome B. Johnson ${ }^{\mathrm{a}, *}$, James D. Cargile ${ }^{\mathrm{b}}$ and Donald M. Smith ${ }^{\mathrm{b}}$ \\ ${ }^{a}$ USA Engineer Research and Development Center, Cold Regions Research and Engineering Laboratory, PO Box \\ 35170, Ft. Wainwright, AK 99703, USA \\ ${ }^{\mathrm{b}}$ USA Engineer Research and Development Center, Geotechnical and Structures Laboratory, 3909 Halls Ferry \\ Road, Vicksburg, MS 39180, USA
}

Received 7 November 2005

Revised 20 November 2006

\begin{abstract}
A physical model of low-velocity probe penetration is developed to characterize soil by type, strength, maximum compaction, and initial density using Newton's second law to describe the processes controlling probe momentum loss. The probe loses momentum by causing soil failure (strength), accelerating and compacting soil around the probe (inertia), and through frictional sliding at the probe/soil interface (friction). Probe geometry, mass, and impact velocity influences are incorporated into the model. Model predictions of probe deceleration history and depth of penetration agree well with experiments, without the need for free variables or complex numerical simulations.
\end{abstract}

Keywords: Soil strength, soil density, soil compaction, impact penetrator deceleration, soil properties

\section{Introduction}

Determining the near-surface soil properties (strength, density, compaction, type or texture) of planets, comets, and inaccessible or denied regions on Earth is of high interest to planetary scientists and military planers. Planetary scientists are interested in understanding the formation processes of planets and comets and in planning for landed missions, while military planners and engineers have a need to know the state-of-the-ground in regions where military operations may be conducted, but are too remote or dangerous to visit. Analysis of low-velocity probe deceleration (equivalent to a negative acceleration) is the most practical method to remotely determine ground state. In this paper, we present a theory of low-velocity probe penetration in soil that is based on a physical understanding of the processes observed from a variety of experimental results. Derived soil properties include strength, density, maximum compaction, and type or texture from which it may be possible to estimate the soil resilient modulus for subgrade soils, an important parameter for pavement design and evaluation.

The lack of a physics based interpretation theory, where each of the acting mechanisms is identified and determined separately, limits the accuracy of interpreting low-velocity probe measurements. Interpretation of probe measurements can be accomplished using numerical methods or penetration theories that can be purely empirical, empirical with physical elements, or purely physical. Numerical approaches using finite-element, finite-difference, smoothed particle hydrodynamics or other methods solve the continuity, momentum and energy balance equations of continuum mechanics in conjunction with an appropriate constitutive representation for the target soil properties. These first-principles techniques can accurately simulate a penetration event when the target and probe properties are well defined, but backing out soil properties from a probe deceleration record is difficult because the effects

\footnotetext{
*Corresponding author. Tel.: +1 907353 5179; Fax: +1 907353 5142; E-mail: Jerome.B.Johnson@erdc.usace.army.mil.
} 

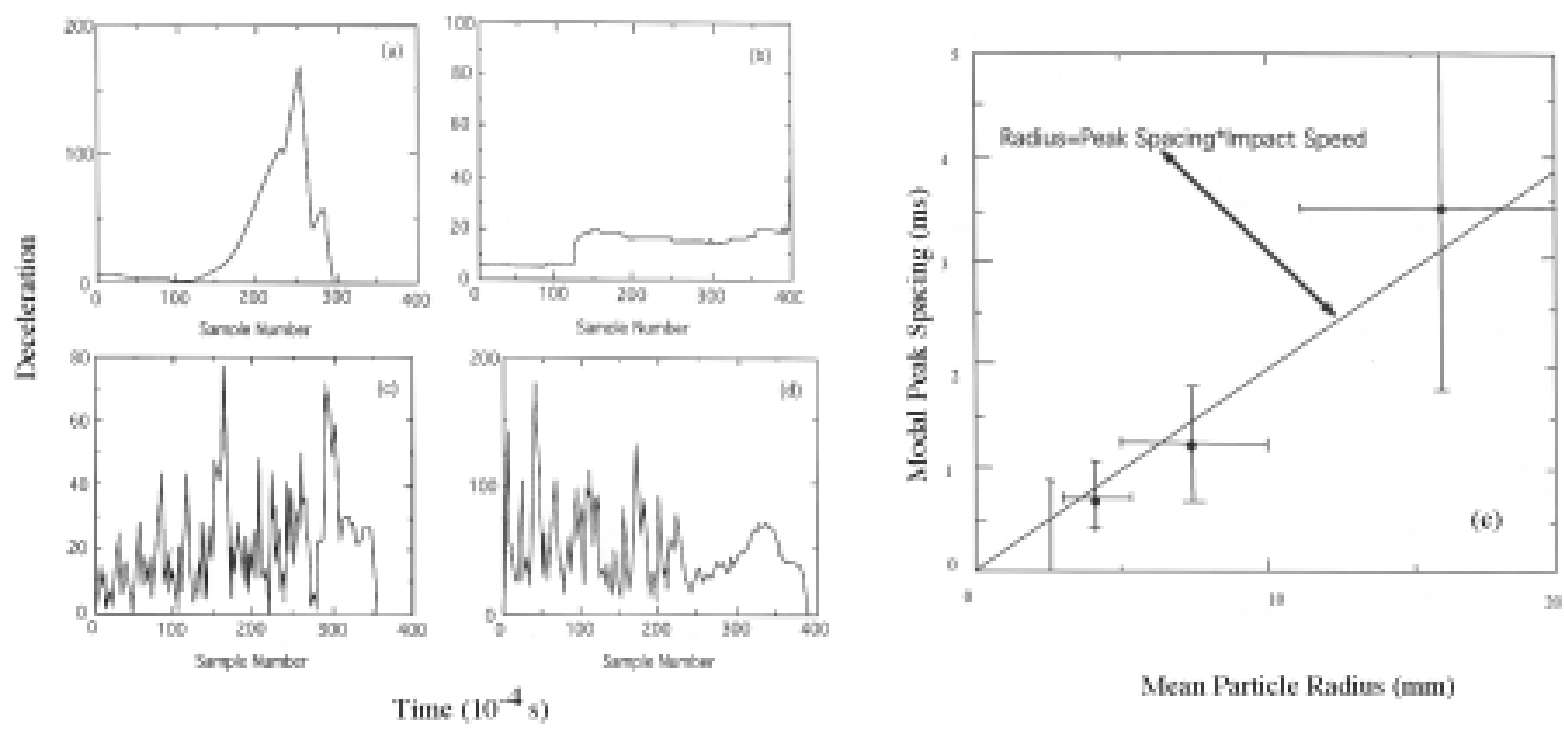

Fig. 1. Penetrator deceleration records for sand (a), Clay (b), fine gravel ( $8 \mathrm{~mm})(\mathrm{c})$, medium grade gravel $(15 \mathrm{~mm})(\mathrm{d})$. The time between peaks for gravel as a function of mean particle radius (e) (Data re-plotted from [8]).

of soil behavior, inertia, and probe properties are mixed during computation. Estimates of soil properties require computationally intensive, non-unique, "guess and check" methods.

Empirical models are based on experimental correlations of parameters such as penetration depth, crater volume, impact velocity or momentum, probe geometry, or other measurable quantities and generally offer little insight to the physical processes that are occurring [1,2]. Empirical models with physical elements are generally analytical models that provide correlations like those from empirical studies, but introduce relations between parameters of the system on the basis of physical requirements. The best known such relation is a resistive force that is a function of the projectile velocity where the coefficients are associated with frictional and added mass effects [1]. While better than empirical models, these models are also not well suited for inferring target properties from penetration measurements because their coefficients are essentially free parameters set through correlation with experiments.

A purely physical approach uses experimental observations to guide the formulation of an ideal model of the processes that produce resistance forces on a probe. Existing physical models generally over simplify the deformation geometry and do not include all relevant physics [3-6]. Comparisons of data with theoretical predictions using current physical models indicate that the models underestimate the importance of added mass (compacted soil that moves with the probe) effects, by a factor of five [7].

Identification of soil type can be accomplished by examining the unique probe deceleration-depth curve pattern that occurs for different soil types (Fig. 1). In frictional soils, probe deceleration increases with penetration depth to a peak value and then drops rapidly to zero because of increasing soil strength and density with depth. Probe deceleration in cohesive soils initially increases from zero to a constant value when soil strength is constant as a function of penetration depth. Coarse materials will cause rapidly varying deceleration spikes whose amplitude and frequency are related to particle size and spacing [8]. Mixed materials, consisting of coarse particles imbedded in either cohesive or frictional materials, produce a probe deceleration signal that contains aspects of each of the constituent material types.

Available data indicate that at ordinance velocities penetration resistance forces can arise from the failure and compaction of the initially undeformed soil, added mass effects, soil particle comminution, friction between the probe and soil, and failure within the compacted soil region $[1,9,10]$. Furthermore, the shape of the probe tip determines the geometry of the zone of compacted soil around a probe and partition of importance of the controlling mechanisms.

For penetrators with small cone half-angles $\left(\theta<45^{\circ}\right)$, mass compaction is generally normal to the surface of the cone producing a conically shaped compaction surface around the conical tip ([11] and the current study). However, 
for larger cone half-angles, shear stresses in the zone of compacted soil may cause a shear failure through the compacted soil producing a conical plug of soil with a cone half-angle of about $45^{\circ}$ in front of the penetrator [10]. When this occurs, quantitative interpretation of the penetrator deceleration signal is not practical.

\section{Theory of low-velocity penetrator deceleration in soil}

The change in momentum of a penetrator impacting soil is described using Newton's second law stated as

$$
-\frac{d P_{p}}{d t}=\iint_{s_{p}}\left(\frac{d P_{\mu}}{d t}+\frac{d P_{I}}{d t}\right) d s+\iint_{s_{c}}\left(\frac{d P_{\sigma}}{d t}\right) d s
$$

where the change in momentum of the penetrator with time is $d P_{p} / d t$ and the friction force per unit surface area of the penetrator is $d P_{\mu} / d t$. The force per unit area due to the inertia and strength of the impacted soil is given by $d P_{I} / d t$ and $d P_{\sigma} / d t$. Friction and inertia forces act against the penetrator surface area, $S_{p}$, while soil failure occurs against the outer surface of the compacted soil, $S_{c}$, which define the integration surface areas for Eq. (1).

Momentum change of a penetrator from soil inertia occurs as fractured or disaggregated particles, created during the soil failure process, are accelerated and compacted by the penetrator until reaching a final locked density and is given by

$$
\iint_{s_{p}} \frac{d P_{I}}{d t} d s=\sin \theta \iint_{s_{p}}\left(\frac{d P_{I}}{d t}\right)_{n} d s=\sin \theta \iint_{s p}\left(v_{c} \frac{d m_{c}}{d t}+m_{c} \frac{d v_{c}}{d t}\right) d s .
$$

where $\iint_{s_{p}}\left(\frac{d P_{I}}{d t}\right)_{n} d s$ refers to the inertia momentum change directed normal to the penetrator surface and $\iint_{s_{p}} \frac{d P_{I}}{d t} d s$ is the inertia momentum change directed along the penetrator axis. The mass of the compacted soil is $m_{c}$ and $v_{c}=v_{p} \sin \theta$ is the velocity of the compacted soil normal to the penetrator surface were $\theta$ is the cone half-angle and $v_{p}$ is the penetrator velocity. The velocity of the compacted soil is assumed to be constant with respect to $S_{p}$ for the derivation given in this paper. This will be changed to account for the decrease in particle velocity as a function of radial distance from the penetrator surface in a future version of the model.

In the model, soil strength is represented as cohesive or frictional. Cohesive soil strength produces a constant resistance to momentum as a function of depth, but frictional soils (e.g., a Mohr-Coulomb material) produce an increasing resistance to penetration with depth because of the weight of overlying layers. Momentum change from soil strength is given by

$$
\iint_{s_{c}} \frac{d P_{\sigma}}{d t} d s=\sin \theta \iint_{s_{c}}\left(\frac{d P_{\sigma}}{d t}\right)_{n} d s=\sin \theta \sigma_{s} A_{c}
$$

where $A_{c}$ is the surface area at the interface between the compacted and uncompacted soil and

$$
\sigma_{s}=\sigma_{c}+K_{0} Q \tan \varphi \text {. }
$$

where $\sigma_{c}$ is the cohesive strength of the soil, $K_{0}$ is a ratio of horizontal to lateral pressure, $Q$ is the horizontal pressure, and $\varphi$ is the soil internal friction angle. $Q$ is a depth dependent function given by

$$
Q=\rho_{0} g Z_{p}
$$

where $\rho_{0}$ is the soil initial density as a function of depth $Z_{p}, g$ is gravitational acceleration, and $Z_{p}$ is the depth of penetration. The strength term is of the form of a Mohr-Coulomb material, but is used in our formulation to fit the soil strength derived from the penetrator analysis.

Frictional sliding acts along the interface boundary between the penetrator and compacted soil. The momentum change due to friction depends on the normal force acting on the cone surface, which is the sum of the momentum change from inertia and strength, and the coefficient of friction, $\mu$. The friction momentum change directed axially along the penetrator is

$$
\iint_{s_{p}} \frac{d P_{\mu}}{d t} d s=\mu \cos \theta\left[\iint_{s_{p}}\left(\frac{d P_{I}}{d t}\right)_{n} d s+\iint_{s_{c}}\left(\frac{d P_{\sigma}}{d t}\right)_{n} d s\right]
$$




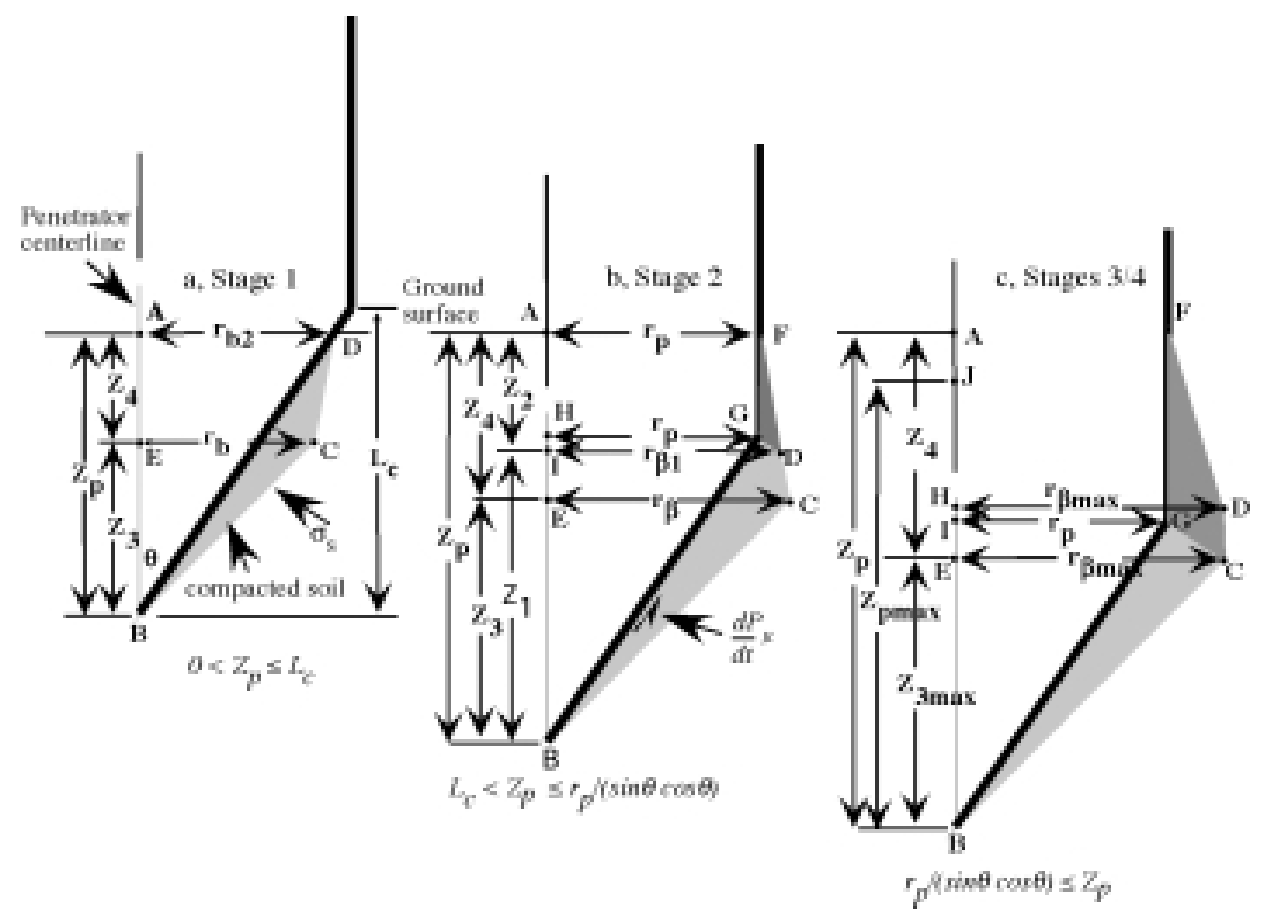

Fig. 2. The four stages of penetration. Stages $3 \& 4$ are shown together in (c) since the only difference between them is that the penetrator aftbody is not completely beneath the soil surface in stage 3 , but is completely buried in stage 4 .

Probe penetration into soil occurs in four stages. In stage 1 the cone first engages the soil compacting it and adding its mass to the penetrator nose. In stage 2, the cone becomes buried in the soil and some of the compacted soil begins to slough off of the cone. During stage 3 the probe aftbody is entering the soil and full burial of the probe aftbody defines the start of stage 4 penetration (Fig. 2).

Using the formulations for momentum change, strength, and friction given in Eqs (2), (3), and (6) and calculating the compaction zone around the cone in terms of $Z_{p}$ for each of the four stages of probe penetrations gives

$$
\begin{aligned}
-\frac{d P_{p 1}}{d t}= & (\sin \theta+\mu \cos \theta)\left(\rho_{f} \sin \theta B_{B C D 1} Z_{p}^{2}\left(3 v_{p 1}^{2}+\frac{d v_{p 1}}{d t} Z_{p}\right)+\sigma_{s} A_{B C D 1}\right) \\
-\frac{d P_{p 2}}{d t}= & (\sin \theta+\mu \cos \theta)\left[\rho_{f} \sin \theta\left(v_{p 2}^{2}\left(F_{B C D G 2}+F_{D F G 2}\right)+\frac{d v_{p 2}}{d t} V_{B C D G 2}\right)+\sigma_{s} A_{B C D 2}\right] \\
& +\mu \sigma_{s} \pi r_{p}^{2}\left(Z_{p}-L_{c}\right) \\
-\frac{d P_{p 3}}{d t}= & (\sin \theta+\mu \cos \theta)\left[\rho_{f} \sin \theta\left[v_{p 3}^{2} \pi r_{p}^{2}\left(\frac{1}{\beta}-1\right)+\frac{d v_{p 3}}{d t} V_{B C G 3}\right]+\sigma_{s} A_{B C 3}\right] \\
& +\mu \sigma_{s} \pi r_{p}^{2}\left(Z_{p}-L_{c}\right) \\
-\frac{d P_{p 4}}{d t}= & (\sin \theta+\mu \cos \theta)\left[\rho_{f} \sin \theta\left[v_{p 4}^{2} \pi r_{p}^{2}\left(\frac{1}{\beta}-1\right)+\frac{d v_{p 4}}{d t} V_{B C G 3}\right]+\sigma_{s} A_{B C 3}\right]+\mu \sigma_{s} \pi r_{p}^{2} L_{p}
\end{aligned}
$$

The subscript numbers indicate the penetrator stage for which the equation is valid, definitions for the parameters $B_{B C D 1}, A_{B C D 1}, F_{B C D G 2}, F_{D F G 2}, V_{B C D G 2}, A_{B C D 2}, V_{B C G 3}$, and $A_{B C 3}$ are given in Appendix A. The $V$ and $A$ parameters represent volume and area of the compacted soil over the region defined by the subscripting letters as shown in Fig. 2. The average compaction of the soil around the penetrator is given by $\beta=1-\frac{\rho_{0}}{\rho_{f}}$, where $\rho_{f}$ is the average final density of soil in the zone of compaction (Fig. 2). 
Table 1

Laboratory determined properties of target soil

\begin{tabular}{cccccc}
\hline $\begin{array}{c}\rho_{0} \\
\left(\mathrm{~kg} / \mathrm{m}^{3}\right)\end{array}$ & $\begin{array}{c}\text { Max. } \\
\text { density, } \rho_{\max } \\
\left(\mathrm{kg} / \mathrm{m}^{3}\right)\end{array}$ & $\begin{array}{c}\text { Fractional } \\
\text { max. } \\
\text { density }\end{array}$ & $\begin{array}{c}\beta \\
\left(1-\rho_{0} / \rho_{\max }\right)\end{array}$ & $\begin{array}{c}\text { Moisture } \\
(\%)\end{array}$ & $\begin{array}{c}\text { Friction } \\
\text { angle } \\
\left({ }^{\circ}\right)\end{array}$ \\
\hline 1730 & $1778-1810$ & $0.67-0.68$ & $0.03-0.04$ & $2-4$ & 45 \\
\hline
\end{tabular}

Table 2

Calculated final and fractional densities of the compacted soil and depths of penetration as a function of $\beta$ using initial density and friction angle input data from Table 1

\begin{tabular}{lccc}
\hline \multicolumn{1}{c}{$\beta$} & $\rho_{f}\left(\mathrm{~kg} / \mathrm{m}^{3}\right)$ & Fractional final density & Depth of penetration (meters) \\
\hline 0.26 & 2323 & 0.87 & 1.04 \\
0.2 & 2162 & 0.81 & 0.81 \\
0.14 & 2002 & 0.75 & 0.75 \\
0.1 & 1922 & 0.72 & 0.72 \\
0.06 & 1842 & 0.69 & 0.31 \\
$\mathbf{0 . 0 1 6}$ & $\mathbf{1 7 5 8}$ & $\mathbf{0 . 6 6}$ & $\mathbf{0 . 2 9}$ \\
\hline
\end{tabular}

Recursion relations derived from Eqs (7)-(10) for calculating the penetrator forward velocity $\left(v_{p+}\right)$ as a function of time from the current penetrator velocity $\left(v_{p}\right)$ are given for penetration stages $1-4$ by

$$
\begin{aligned}
& v_{p 1+}=v_{p 1}-\frac{(\sin \theta+\mu \cos \theta)\left[\rho_{f} \sin \theta B_{B C D 1} z_{p}^{2} 3 v_{p 1}^{2}+\sigma_{s} A_{B C D 1}\right]}{M_{p}+(\sin \theta+\mu \cos \theta) \rho_{f} \sin \theta B_{B C D 1} Z_{p}^{3}} \Delta t \\
& v_{p 2+}=v_{p 2}-\frac{(\sin \theta+\mu \cos \theta)\left[\rho_{f} \sin \theta v_{p 2}^{2}\left(F_{B C D G 2}+F_{D F G 2}\right)+\sigma_{s} A_{B C D 2}\right]+\mu \sigma_{s} \pi r_{p}^{2}\left(Z_{p}-L_{c}\right)}{M_{p}+(\sin \theta+\mu \cos \theta) \rho_{f} \sin \theta V_{B C D G 2}} \Delta t \\
& v_{p 3+}=v_{p 3}-\frac{(\sin \theta+\mu \cos \theta)\left[\rho_{f} \sin \theta\left[v_{p 3}^{2} \pi r_{p}^{2}\left(\frac{1}{\beta}-1\right)\right]+\sigma_{s} A_{B C 3}\right]+\mu \sigma_{s} \pi r_{p}^{2}\left(Z_{p}-L_{c}\right)}{M_{p}+(\sin \theta+\mu \cos \theta) \rho_{f} \sin \theta V_{B C G 3}} \Delta t \\
& v_{p 4+}=v_{p 4}-\frac{(\sin \theta+\mu \cos \theta)\left[\rho_{f} \sin \theta\left[v_{p 4}^{2} \pi r_{p}^{2}\left(\frac{1}{\beta}-1\right)\right]+\sigma_{s} A_{B C 3}\right]+\mu \sigma_{s} \pi r_{p}^{2} L_{p}}{M_{p}+(\sin \theta+\mu \cos \theta) \rho_{f} \sin \theta V_{B C G 3}} \Delta t
\end{aligned}
$$

where $\Delta t$ is the time step and $M_{p}$ is the penetrator mass.

\section{Comparison of model calculations with experiment}

Depth of penetration experiments were conducted using a spring-loaded "gun" to fire $0.051-\mathrm{m} \mathrm{diameter,} \mathrm{8-kg}$ projectiles with $30^{\circ}$ half-angle conical tips at speeds of up to $15 \mathrm{~m} / \mathrm{s}$ for comparison with model predictions. The properties of the target soil shown in Table 1 were characterized using standard geotechnical testing methods [12]. The maximum density for the soil was determined from a dynamic compaction test and is the maximum density that the soil can achieve without comminution of soil grains or vibration densification. The fractional maximum density range was determined by dividing the maximum density by the mineral soil particle density $\left(2660 \mathrm{~kg} / \mathrm{m}^{3}\right)$.

The initial tests were conducted without a mounted accelerometer on the projectile. Consequently, comparison is to depth of penetration with model calculations. The initial soil density and soil internal friction angle from Table 1 were used as initial input data for the model. The average compaction coefficient $(\beta)$ was varied until the model predicted depth of penetration of an 8-kg, 0.051-m-diameter penetrator with a $30^{\circ}$ conical half-angle and an initial speed of $15.2 \mathrm{~m} / \mathrm{s}$ matched the measured depth of penetration $(0.29 \mathrm{~m})$ of the actual penetrator. A soil $\mathrm{K}_{0}=0.5$ was used for the strength factor, which is generally considered to be the maximum lateral pressure exerted by a soil, and the friction coefficient $\mu=0.3$ was used for the steel/sand interface. 


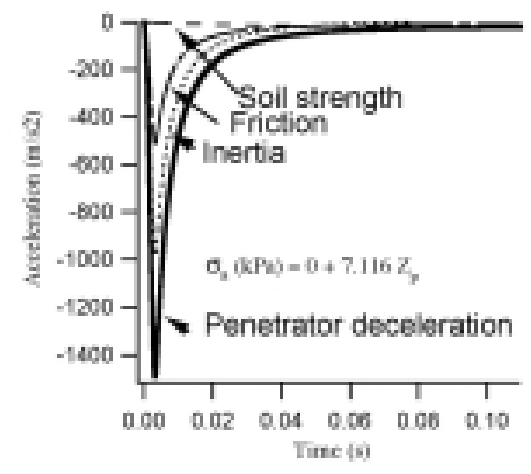

Fig. 3. Penetrator deceleration (negative acceleration) for an $8-\mathrm{kg}$ penetrator with $3 \Theta$ cone half-angle and initial velocity of $15.2 \mathrm{~m} / \mathrm{s}$. Initial soil conditions are given in Table 1 and $\beta=0.016$.

The calculated total penetrator acceleration and the partition of the acceleration into the contributions from soil strength, friction between the penetrator and soil, and inertia associated with soil particle acceleration during soil compaction are shown in Fig. 3 for the case of $\beta=0.016$. The table of calculated depth of penetration results and the associated final and fractional final densities (the fractional density is the ratio of final density to solid soil mineral density $\left(\rho_{f} / \rho_{s}\right)$ for a range of $\beta$ values from $0.26-0.016$ are given in Table 2 .

The results shown in Fig. 3 indicate that soil strength contributed very little to the overall penetrator deceleration (negative acceleration). This is reasonable since the strength of a frictional soil, without cohesion, depends strongly on lateral soil pressure (Equations (4) and (5)), which is zero at the surface and very low at the shallow depths associated with our penetrator tests. The majority of penetrator deceleration was caused by soil inertia with some additional contribution from friction. The model calculated penetrator deceleration shown in Fig. 3 has the same general shape as the deceleration for a frictional soil shown in Fig. 1a. This implies that the model physics is capturing the general characteristics of penetrator deceleration in frictional soils.

The results given in Table 2 indicate that the model calculated depth of penetration matches the experimental values for $\beta=0.016$ with corresponding average final density of $1758 \mathrm{~kg} / \mathrm{m}^{3}$ (the boldfaced numbers). The average final density compares well with measured maximum densities for the soil of $1778-1810 \mathrm{~kg} / \mathrm{m}^{3}$ (Table 1) indicating that given the initial soil density, soil internal friction angle, and penetrator depth of penetration the model accurately predicts the compaction density. Conversely, the depth of penetration can be predicted when the final compacted density is known.

The ability of the model to calculate the correct form of penetrator deceleration for a frictional soil (compare Fig. 3 with Fig. 1a) and accurately predict the depth of penetration using actual soil properties without arbitrary fit parameters indicates that the model contains reasonable physically based algorithms. This is the hallmark of a physically representative model that is needed to interpret penetrator performance in soil to determine soil properties.

\section{Soil properties from low-velocity probe deceleration}

The limits of using a low-velocity probe to determine soil properties depend directly on the accuracy of the physical penetration model, deceleration measurement resolution, and our understanding of the relationship between soil properties and probe deceleration. It is not possible to accurately characterize soil properties without a physical model because of the combination of effects from the probe (geometry, cone half-angle, mass, impact velocity) and soil (initial density, strength, compaction, probe/soil friction, particle size) that affect probe deceleration. To extract soil properties information from the probe deceleration record requires that the effects of the probe and soil must be distinguishable from each other. The physical model can separately account for the probe effects, which are uniquely known, and inertia, allowing interpretation efforts to focus on the soil. However, even with an accurate physical model, it is not possible to uniquely characterize soil properties without further reducing the number of unknown variables by bringing additional information into the interpretation process. This includes using the unique 
penetrator deceleration histories associated with different types of soils, as shown in Fig. 1, to identify soil type. It also includes using the maximum fractional density of random close packed particles, 0.6 , to help constrain the compaction coefficient.

From the results shown in Fig. 1, it is apparent that by observing the general character of the probe deceleration record it is possible to identify the general soil type, hence its texture. A cohesionless frictional soil produces a continuously increasing probe deceleration with depth, as the soil strength increases with increasing overburden, to a maximum value and then rapidly decreases to zero as the probe comes to rest (Fig. 1a). Probe deceleration for a cohesive soil increases to a constant value until the probe stops (Fig. 1b). The magnitude of the cohesive strength is indicative of the type of cohesive soil (clay, wet sand or silt, sandstone). As soil coarseness increases, or small particle gravels are encountered, acceleration of individual particles can be seen in the probe deceleration record (Fig. 1c and d). The size and mass of the particles are related to the magnitude and spacing of the deceleration peaks of the penetrator (Fig. 1c, d, and e).

A knowledge of particle packing behavior can be used to limit the range of expected maximum fractional density of compacted soil to slightly more than 0.6 , the maximum random close packing of spheres, or less unless particle comminution or vibration occurs [13]. Evidence of this limit on soil particle packing is given in the test results of Table 1 for the fractional maximum density range of the soils used in the current tests and the model derived maximum fractional density of 0.66 from the dynamic test results (Table 2).

Resilient modulus of subgrade soils is an important factor in pavement design and mobility analyses that are typically evaluated using simple empirical relationships with CBR values. Studies have indicated that both the resilient modulus and CBR are related to the undrained soil shear strength, hence to each other [14-16]. This implies that a relationship between probe derived soil strength and the resilient modulus and CBR should exist, providing a pathway from soil properties derived from low-velocity probe measurements to the resilient modulus and CBR.

\section{Conclusions}

A physically based model of low-velocity probe penetration into soil was developed using the essential factors that control probe deceleration through transference of probe momentum to the soil. Probe momentum is lost to soil failure processes at the outer edge of the zone of compacted soil around the probe (soil strength), compaction and acceleration of the soil particles after failure (soil inertia), and sliding between the probe and surrounding soil (friction).

The effect of soil strength is to produce a constant momentum resistance for cohesive soils and an increasing momentum resistance with depth for cohesionless frictional soils. Soil inertia momentum resistance increases with the square of the probe velocity, the probe deceleration, the final compacted soil density, and with a decreasing soil compaction coefficient. Friction between the probe and soil depends on the coefficient of friction between the probe and soil, the normal pressure acting against the probe, and the area of probe surface in contact with the soil. The normal pressure against the probe is dominated by momentum change from soil strength and inertia. All of the momentum loss factors are affected by probe geometry including cone half-angle and the probe radius and length. This produces a set of coupled equations that describe each of the four stages of penetration (cone entry, cone burial, aftbody entry, and aftbody burial), which are solved through recursion relations.

The ability of the model to calculate the correct form of penetrator deceleration for a frictional soil (compare Fig. 3 with Fig. 1a) and to accurately predict the depth of penetration using actual soil properties without arbitrary fit parameters indicates that the model contains reasonable physically based algorithms. The soil maximum fractional density derived from matching model-calculated depth of penetration with measurement agreed to within a few percent. The values measured in the laboratory demonstrate the constraints that a physical model places on the range of possible soil parameters that can be used when fitting model results with experiment. Probe deceleration analysis can provide information on soil type, strength, compaction, and a qualitative estimate of grain sizes. Relationships between undrained shear strength and the California Bearing Ratio test and resilient modulus may provide a pathway to interpreting soil properties derived from low-velocity probe measurements with subgrade properties that are important for pavement design and evaluation and mobility analyses.

Improvements to the model will require experiments to improve our understanding of near-surface soil failure processes that produce craters and the relationship between soil type and texture on the magnitude and overall deceleration history of a probe. 


\section{Acknowledgements}

We thank E. Berney, K. Jones, and M. Guillet for their help in conducting experiments and developing and implementing a computational version of the theory. The USA Engineer Research and Development Center Military Engineering Basic Research AT22 Work Package supported this work. Permission from the Headquarters, US Army Corps of Engineers, to publish this paper is gratefully acknowledged.

\section{Appendix A: Parameter definitions for Eqs (7)-(14)}

$$
\begin{aligned}
& B_{B C D 1}=\frac{\pi \sin ^{2} \theta}{3}\left[\frac{1}{\beta}-\frac{\sqrt{B}-\sin ^{2} \theta}{\sqrt{B} \cos ^{2} \theta}\right] \\
& A_{B C D 1}=\pi r_{\beta} \sqrt{r_{\beta}^{2}+Z_{3}^{2}}+\pi\left[\left(r_{b 2}+r_{\beta}\right) \sqrt{\left(r_{b 2}-r_{\beta}\right)^{2}+Z_{4}^{2}}\right] \\
& V_{B C D G 2}=\frac{\pi}{3} Z_{3} r_{\beta}^{2}+\frac{\pi}{3}\left(Z_{1}-Z_{3}\right)\left[r_{\beta}^{2}+r_{\beta_{1}}^{2}+r_{\beta} r_{\beta 1}\right]+\frac{\pi}{3} L_{H I}\left[r_{p}^{2}+r_{\beta 1}^{2}+r_{p} r_{\beta 1}\right]-\frac{\pi}{3} L_{c} r_{p}^{2}
\end{aligned}
$$

where $\mathrm{L}_{H I}$ is the distance between reference points $\mathrm{H}$ and $\mathrm{I}$ in Fig. 2 b.

$$
A_{B C D 2}=\pi r_{\beta} \sqrt{r_{\beta}^{2}+Z_{3}^{2}}+\pi\left(r_{\beta 1}+r_{\beta}\right) \sqrt{\left(r_{\beta 1}-r_{\beta}\right)^{2}+\left(z_{1}-z_{3}\right)^{2}}
$$

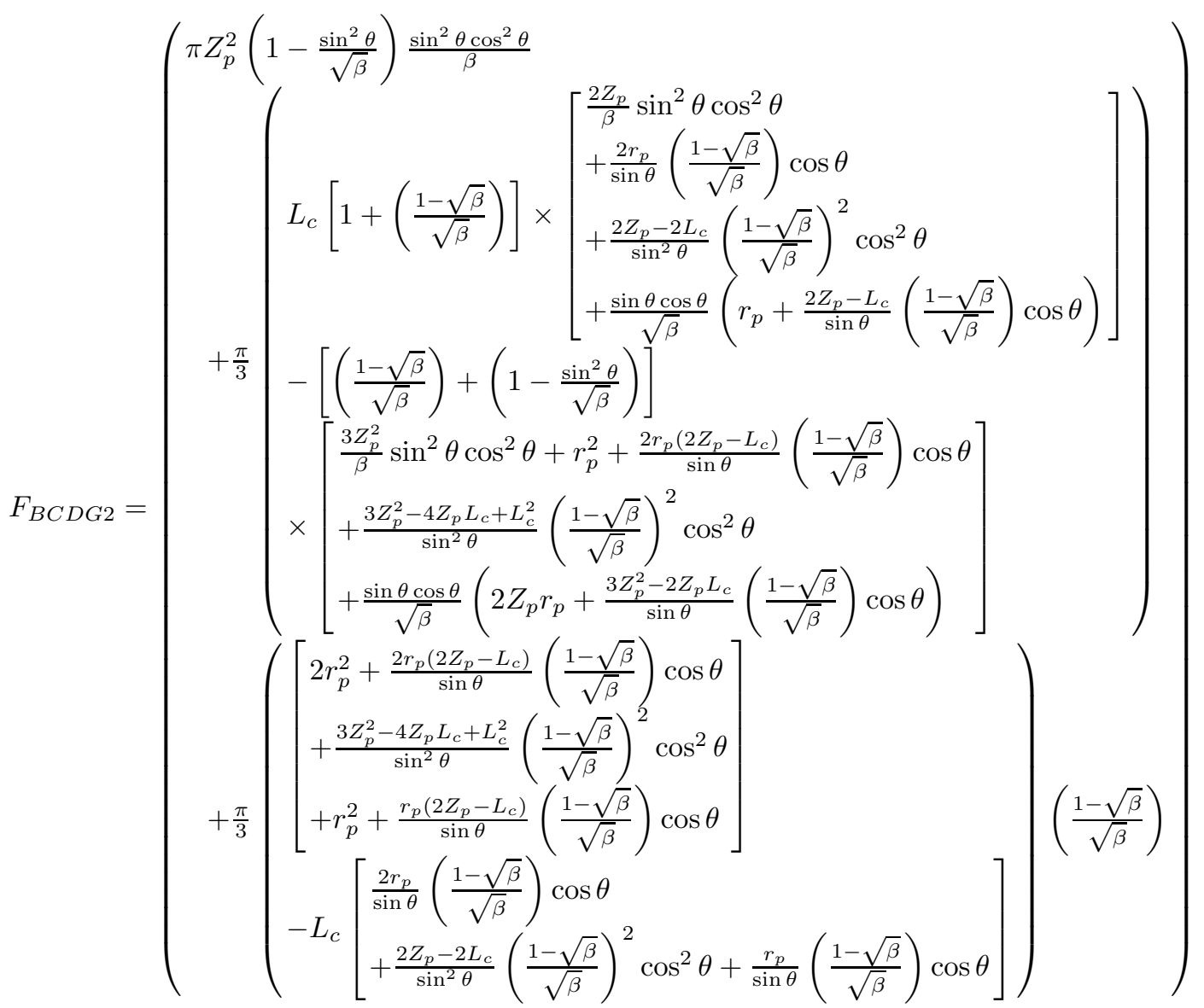




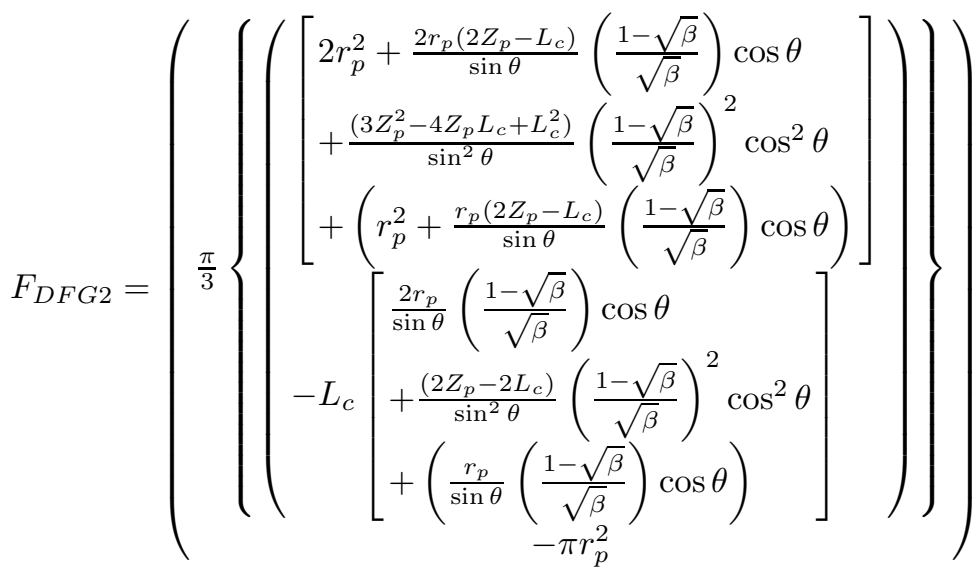

$$
\begin{aligned}
& V_{B C G 3}=\frac{\pi}{3}\left[L_{c}\left(r_{\beta \max }^{2}+r_{\beta \max } r_{p}\right)+Z_{3}\left(r_{p}^{2}+r_{\beta \max } r_{p}\right)\right] \\
& A_{B C 3}=\pi \frac{r_{p}}{\sqrt{\beta}} \sqrt{\frac{r_{p}^{2}}{\beta}+\left(\frac{r_{p}}{\sin \theta \cos \theta}\left(1-\frac{\sin ^{2} \theta}{\sqrt{\beta}}\right)\right)^{2}}
\end{aligned}
$$

\section{References}

[1] M.E. Backman and W. Goldsmith, The mechanics of penetration of projectiles into targets, International Journal of Engineering Science 16 (1978), 1-99.

[2] C.W. Young, Penetration Equations, Sandia National Laboratories Contractor Report SAND97-2426, 1997.

[3] M.J. Forrestal, Penetration into dry porous rock, International Journal of Solids and Structures 22 (1986), 1485-1500.

[4] D.D. Fritton, A standard for interpreting soil penetrometer measurements, Soil Science 105 (1990), 542-551.

[5] M.J. Forrestal and V.K. Luk, Penetration into soil targets, International Journal of Impact Engineering 12 (1992), 427-444.

[6] E.L. Greacen, D.A. Farrel and B. Cockroft, Soil Resistance to Metal Probes and Plant Roots, in: Transactions of the 9th International Congress on Soil Science, Elsevier Publishing Co. and International Society of Soil Science, Adelaide, Australia, 1968, 769-779.

[7] N.I. Kölme, A.J. Ball, G. Kargl, T. Keller, W. Macher, M. Thiel, J. Stöcker and C. Rohe, Impact penetrometery on a comet nucleusinterpretation of laboratory data using penetration models, Planetary and Space Science 49 (2001), 575-598.

[8] R.D. Lorenz, M. Bannister, P.M. Daniell, Z. Krysinski, M.R. Leese, R.J. Miller, G. Newton, P. Rabbetts, D.M. Willett and J.C. Zarmeck, An impact penetrometer for a landing spacecraft, Measurement Science Technology 5 (1994), 1033-1041.

[9] W.A. Allen, E.B. Mayfield and H.L. Morrison, Dynamics of a projectile penetrating sand, Journal of Applied Physics 28 (1957), 370-376.

[10] W.W. Anderson, T.J. Ahrens, A. Gibson, R. Scott and K. Suzuki, Emplacement of penetrators into planetary surfaces, Journal of Geophysical Research 101(E9) (1996), 21137-21149.

[11] W.R. Gill, Influence of compaction hardening of soil on penetration resistance, Transactions for the American Society of Agricultural Engineers 11 (1968), 741-745.

[12] E.S. Berney and D.M. Smith, Mechanical and Physical Properties of ASTM C33 Sand, ERDC/GSL-TR-06-XX, ERDC, Waterways Experiment Station, Vicksburg, 2006

[13] R.M. German, Particle Packing Characteristics, Metal Powder Industries Federation, Princeton 1989.

[14] W.P.M. Black, The calculation of laboratory and in-situ values of California Bearing Ratio from bearing capacity data, Geotechnique 11 (1961), 14-21.

[15] J.M. Duncan and A.L. Buchignani, An Engineering Manual for Settlement Studies, unpublished report, Department of Civil Engineering, University of California, Berkeley, 1976.

[16] M.R. Thompson and Q.L. Robnett, Resilient properties of subgrade soils, Journal of Transportation Engineering, American Society of Civil Engineering 105 (1979), 71-89. 

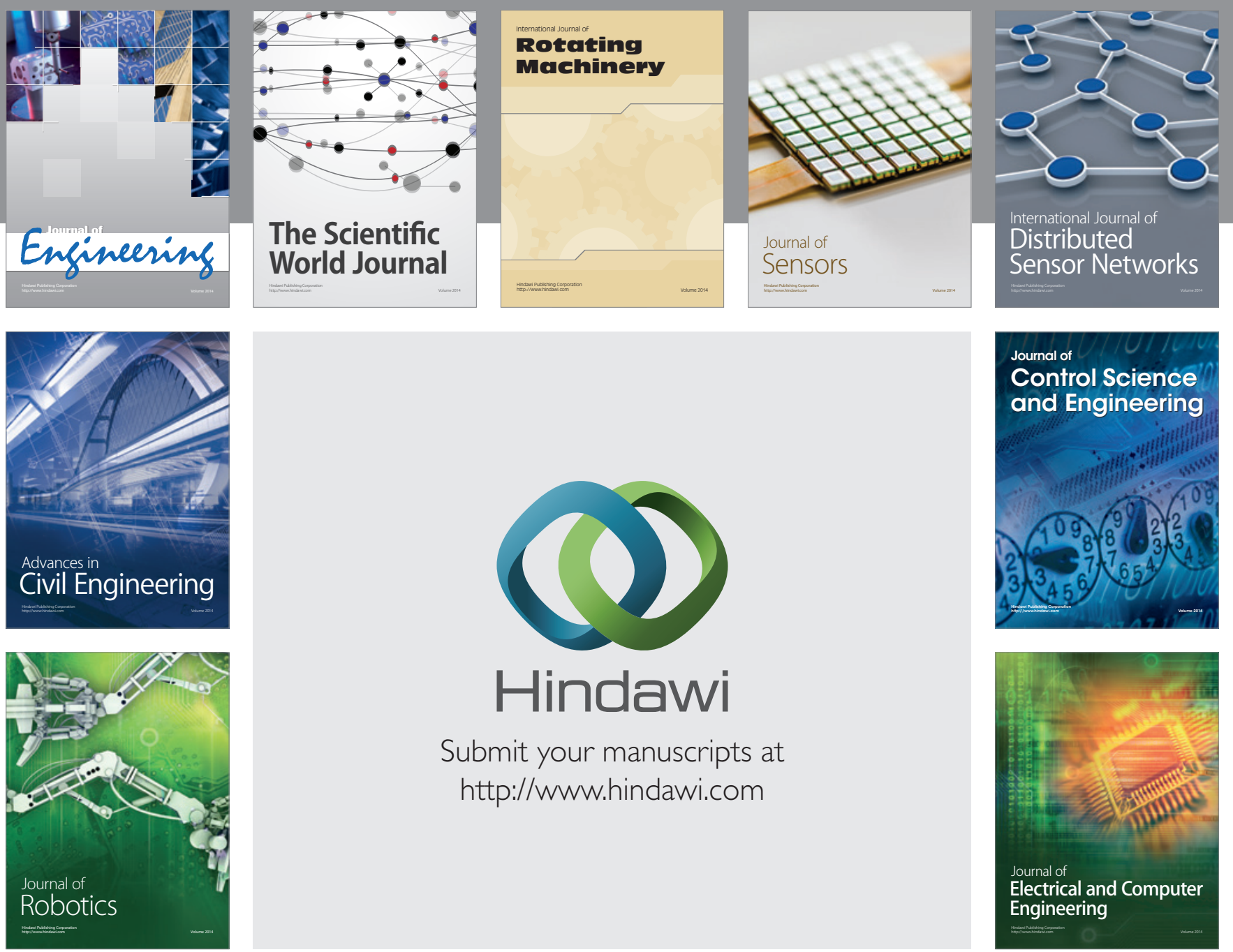

Submit your manuscripts at

http://www.hindawi.com
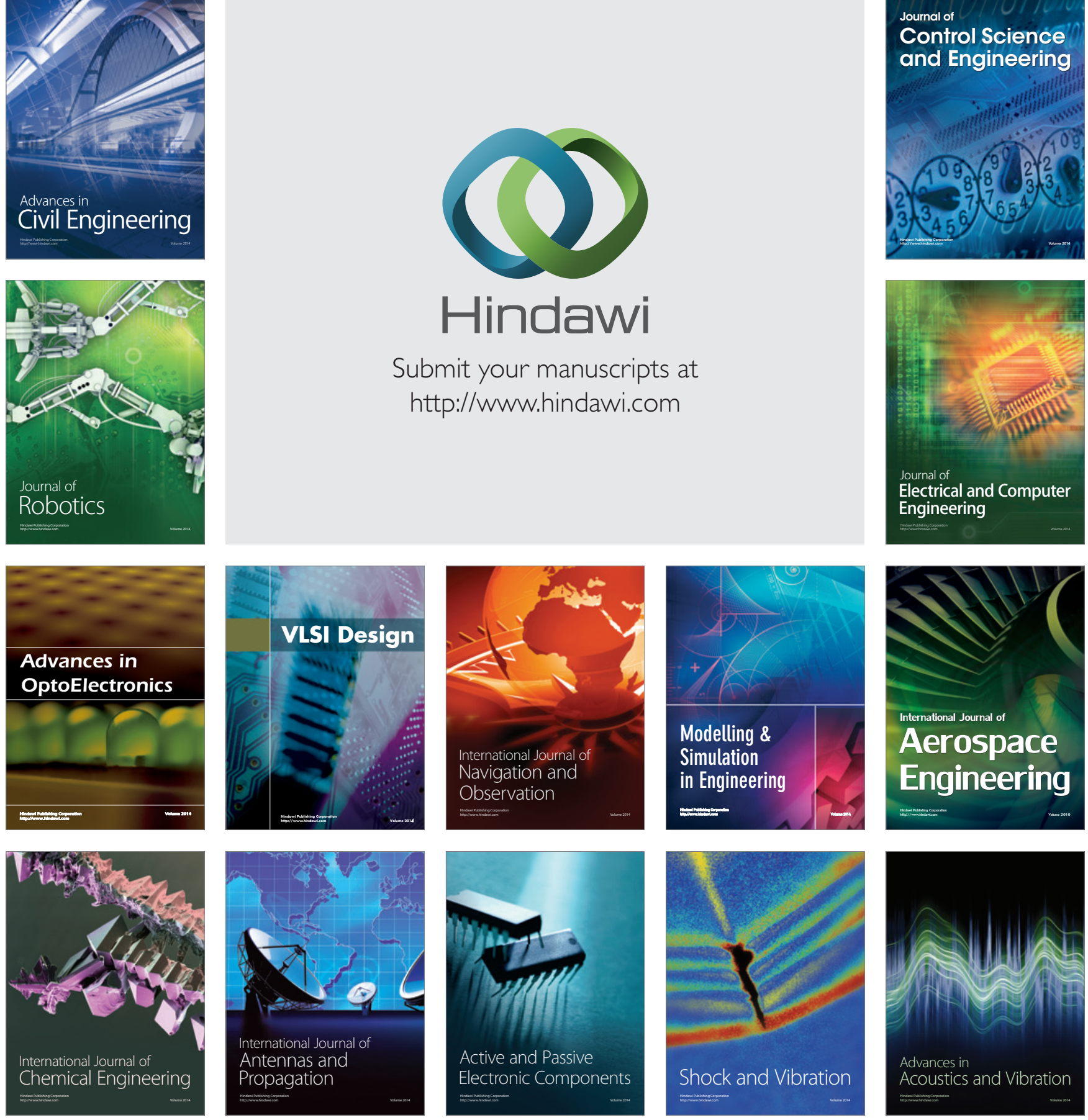